\title{
Studies on Heterosis in Pumpkin (Cucurbita moschata Duch. ex. Poir)
}

\author{
P. Marxmathi ${ }^{1}$, V. Krishnamoorthy ${ }^{1}$ and P. Thankaraj ${ }^{2}$ \\ ${ }^{1}$ Department of Horticulture, ${ }^{2}$ Department of Plant Breeding and Genetics, Agriculture \\ College \& Research institute, Tamil Nadu Agricultural University, \\ Madurai- 625 104, Tamil Nadu, India \\ *Corresponding author
}

\begin{tabular}{|l|}
\hline Ke y w o r d s \\
Pumpkin, Cucurbita \\
moschata, Fruit \\
yield, Heterosis, \\
Carotene
\end{tabular}

\section{A B S T R A C T}

The present study was carried out at Department of Horticulture, Agricultural College and Research Institute, Madurai during 2016-2017. Thirty pumpkin hybrids evolved by crossing six genotypes in diallel mating design were evaluated to study heterosis for quantitative and qualitative traits. The higher significantly negative standard heterosis for days to firsts female flowering was recorded in $\mathrm{P}_{1} \times \mathrm{P}_{2}(-9.03 \%)$ and it was positive in $\mathrm{P}_{2} \mathrm{X}$ $\mathrm{P}_{1}(2.24 \%), \mathrm{P}_{2} \mathrm{X} \mathrm{P}_{5}(5.73 \%), \mathrm{P}_{5} \mathrm{XP}_{2}$ (1.69), $\mathrm{P}_{5} \mathrm{XP}_{6}$ (5.10), $\mathrm{P}_{6} \mathrm{X} \mathrm{P}_{5}$ (5.30). The node to first female flower was significantly positive heterosis was observed in $\mathrm{P}_{2} \mathrm{XP}_{6}(44.71 \%), \mathrm{P}_{3} \mathrm{XP}_{6}$ (21.09\%), $\mathrm{P}_{5} \mathrm{XP}_{6}(21.09 \%), \mathrm{P}_{6} \mathrm{XP}_{3}$ (36.89). The significantly positive heterosis was high in $\mathrm{P}_{4} \mathrm{XP}_{1}(36.24 \%), \mathrm{P}_{5} \mathrm{XP}_{1}(34.31 \%), \mathrm{P}_{4} \mathrm{XP}_{6}(33.49 \%), \mathrm{P}_{4} \mathrm{XP}_{2}(33.01 \%)$, and negative in $\mathrm{P}_{6} \mathrm{XP}_{4}(-4.70 \%)$. The high heterosis for days to first harvest observed in $\mathrm{P}_{5} \mathrm{XP}_{1}(6.46 \%)$ and $\mathrm{P}_{6} \mathrm{XP}_{4}(6.28 \%)$. The high positive significant heterosis observed in $\mathrm{P}_{5} \mathrm{XP}_{1}(32.91 \%)$, for fruit length and negatively in $\mathrm{P}_{3} \mathrm{XP}_{5}(-37.02 \%)$. The fruit diameter heterosis was positively high in $\mathrm{P}_{5} \mathrm{XP}_{2}(5.09 \%)$, negatively in $\mathrm{P}_{3} \mathrm{XP}_{2}(-31.75 \%)$. The number of seeds per fruit significantly positive heterosis in $\mathrm{P}_{5} \mathrm{XP}_{1}(17.80 \%)$, and negative heterosis in $\mathrm{P}_{2} \mathrm{XP}_{6}(-$ $22.15 \%)$. The standard heterosis for fruit weight in $\mathrm{P}_{5} \mathrm{XP}_{1}$ was maximum $(117.44 \%)$ and number of fruits per vine negatively significant in $\mathrm{P}_{5} \mathrm{X} \mathrm{P}_{3}(-13.36 \%)$. The heterosis for fruit yield per vine was high in $\mathrm{P}_{1} \mathrm{XP}_{5}(206.79 \%)$ and $\mathrm{P}_{4} \mathrm{XP}_{2}(182.95 \%)$. The high heterosis for total soluble solids, beta carotene content and dry matter content was found in $\mathrm{P}_{5} \mathrm{XP}_{6}$ $(8.46 \%), \mathrm{P}_{3} \mathrm{XP}_{2}, \mathrm{P}_{2} \mathrm{XP}_{3}(29.17 \%)$ and $\mathrm{P}_{2} \mathrm{XP}_{1}$ (33.77\% respectively).

\section{Introduction}

Pumpkin (Cucurbita moschata Duch. ex. Poir) is one of the important cucurbitaceous vegetable. It is cultivated throughout the tropical regions of India. It has high productivity, nutritive values, good storability and better transport quality. The immature and mature fruits used as vegetable.
In India, pumpkin cultivated in an area of 11,060 hectares with the total production of 2.77 lakh tonnes which have productivity of 25.10 tonnes per hectare during 2014. In Tamil Nadu state, pumpkin grown an area of 1,530 hectares with an annual production of about 37,340 tonnes and productivity of 24.41 tonnes per hectare during 2014 (Saxena and Chander, 2015). Little attention has been given on crop improvement, as compared to 
other cucurbitaceous vegetables. As it is crosspollinated crop, developing new hybrids is possible through heterosis breeding. As the hybrids will have the advantage of higher productivity with uniformity in size and shape.

Pumpkin, being a monoecious and crosspollinated crop, provides an ample scope for exploitation of hybrid vigour. The commercial exploitation of hybrids is easy in pumpkin due to its high seed content and easy seed extraction procedures.

Pumpkin, being a cross pollinated crop exhibits considerable variation for different traits. So far few attempts have been made to improve the local types and number of released varieties available for commercial cultivation is also limited. Hence, the present experiment was carried out to study the heterosis for various growth, yield and quality traits for small fruited type and high yield.

\section{Materials and Methods}

The present study was carried out at Department of Horticulture, Agricultural College and Research Institute, Tamil Nadu Agricultural University, Madurai, during 2016-17. It is located at $09^{\circ} 58^{\prime} 30.5^{\prime \prime} \mathrm{N}$ latitude, $078^{\circ} 12^{\prime} 27.4 \mathrm{E}$ longitude and at an altitude of $158 \mathrm{~m}$ above the mean sea level.

The climate of experimental location is warm. The high temperature prevails during the months of March to August reached the maximum temperature up to $41.9^{\circ} \mathrm{C}$ in April. The temperature drops in December and the low temperature continues up to January, reaching the minimum of $21^{\circ} \mathrm{C}$. The location receives an average annual rainfall of 620.5 $\mathrm{mm}$.

Six pumpkin genotypes viz. P1 (Acc.No. MDU CM23, Thirumangalam local, Madurai district) is high flesh thickness and medium sized fruit, P2 (Acc.No.MDU CM28, Oddanchatram local, Dinddugul district) is small fruited and more number of fruits, P3 (Acc.No. MDU CM29, Harur local, Dharmapuri district) is early days to flowering and small fruited, P4 (Acc.No. MDU CM12, Department of Horticulture, AC \&RI Madurai) is high yield per plant, P5 (Acc.No. MDU CM1, Attur local, Salem district) is more flesh thickness, P6 (Acc.No. MDU CM31, Rajapalayam local, Virudhunagar district) is narrow sex ratio with medium sized fruits were used as parents for crossing programme in all possible combinations adopting full diallel mating design (Doijode and Sullamath, 1983). All the six parents were selected based on the performance in the germplasm screening.

All the $30 \mathrm{~F}_{0}$ seeds along with their parents and standard check $\mathrm{CO} 1$ were raised in Randomized Block Design (RBD) with three replications during December 2016 to evaluate the hybrids. A spacing of $2 \times 2 \mathrm{~m}$ was adopted. Recommended cultural practices and plant protection measures were followed to all the plants. The beta carotene content estimated in the fruits by following the procedure given by Ranganna (1979) and the dry matter content of the fruits measured by following the methods described by AOAC (1975). The data recorded were statistically analysed by using the methodology of Panse and Sukhatme (1967). The standard heterosis formed more emphasis because of more practical values than the relative heterosis and heterobeltiosis estimation. Expression of heterosis even to a small magnitude for individual component character is a desirable factor (Hathcock and David, 1973). The estimation of standard heterosis done by (F1-SP/SP) X 100. Where $\mathrm{F} 1$ is mean of F1, SP is mean value of standard variety. Significance of heterosis was tested by using error mean square as suggested by Turner (1953).

Five plants were tagged in each hybrid and 
parents in each replications and biometrical observations were recorded from the tagged plants. In the present investigation, the heterosis of direct and reciprocal cross combinations derived from the six genetically divergent parents through diallel mating design and it was estimated over mid parent, better parent and standard check variety. Negative heterosis was considered to be better for some of the six characters studied viz., days to first female appearance, nodes to first male flower, sex ratio, small fruit weight, while positive heterosis was considered to be desirable for the remaining traits viz., flesh thickness, number of fruits per vine, fruit weight, fruit yield per vine, total soluble solids, beta carotene content and dry matter content.

\section{Results and Discussion}

\section{Quantitative traits}

The results of the study reveals that the estimates of standard heterosis showed a range of $-9.03\left(\mathrm{P}_{1} \times \mathrm{P}_{2}\right)$ to $5.73\left(\mathrm{P}_{2} \times \mathrm{P}_{5}\right)$. Among thirty hybrids, five expressed significantly positive heterosis, among the five, the hybrid $\mathrm{P}_{2} \times \mathrm{P}_{5}$ (5.73 per cent) recorded the highest value. Significant and negative standard heterosis for days to first female flower was exhibited $\mathrm{P}_{1} \times \mathrm{P}_{2}$ (-9.3). This may due to the dominant alleles present in $\mathrm{P}_{1}$, and $\mathrm{P}_{2}$ resulted heterotic expression in the $F_{1}$. This result confirmed the findings of Doijode (1994) in pumpkin.

Significant and positive standard heterosis alone found and there was no negative effect for desirable direction for first male flower node and it was in the range of $-12.16\left(\mathrm{P}_{4} \times \mathrm{P}_{2}\right)$ to $44.71\left(\mathrm{P}_{2} \times \mathrm{P}_{6}\right)$. Among thirty hybrids, only six hybrids expressed significant heterosis. The highest heterotic value was recorded in $\mathrm{P}_{2}$ x $\mathrm{P}_{6}$ (44.71 per cent) followed by $43.44\left(\mathrm{P}_{3} \mathrm{X}\right.$ $\mathrm{P}_{6}$ ). It may be due to additive gene action.
This was supported by Anupam et al., (2017) in bottle gourd.

Sex ratio showed a range from $-15.83\left(\mathrm{P}_{6} \times \mathrm{P}_{2}\right.$, $\left.\mathrm{P}_{6} \times \mathrm{P}_{3}\right)$ to $36.24\left(\mathrm{P}_{4} \times \mathrm{P}_{1}\right)$. Among thirty hybrids, one hybrid recorded negative and significant standard heterosis. The highest heterotic expression was recorded in $\mathrm{P}_{6} \times \mathrm{P}_{4}$ (36.24 per cent). It may due to additive gene action. Muthaiah et al., (2017) in ridge gourd were reported similar results. Days to harvest heterosis showed a range of $-3.48\left(\mathrm{P}_{2} \times \mathrm{P}_{5}\right)$ to $6.46\left(\mathrm{P}_{5} \times \mathrm{P}_{1}\right)$. Among thirty hybrids, four were significantly positive and there were no negatively significant values. The highest heterotic expression was recorded in $6.46\left(\mathrm{P}_{5} \mathrm{X}\right.$ $\left.\mathrm{P}_{1}\right)$ followed by $6.28\left(\mathrm{P}_{6} \times \mathrm{P}_{5}\right)$. This result confirmed the findings of Hedau and Sirohi (2006) in pumpkin.

The fruit length estimates of standard heterosis showed a range of from $-37.02\left(\mathrm{P}_{3} \times \mathrm{P}_{5}\right)$ to $32.91\left(\mathrm{P}_{5} \times \mathrm{P}_{1}\right)$. Among thirty hybrids, nine hybrids recorded positive and nineteen recorded negative significant standard heterosis. The crosses $\mathrm{P}_{5} \times \mathrm{P}_{1}(32.98 \%), \mathrm{P}_{1} \times$ $\mathrm{P}_{5}(16.64 \%)$ and $\mathrm{P}_{1} \times \mathrm{P}_{6}(14.18 \%)$ exhibited significant and positive standard heterosis for fruit length and this may be due to partial dominance gene action. The highest negative heterotic expression was recorded in $\mathrm{P}_{3} \times \mathrm{P}_{5}$ (37.02 per cent) followed by $\mathrm{P}_{3} \times \mathrm{P}_{4}(-36.83$ per cent) due to the action of recessive alleles Similar results were reported by Kumar et al., (2010) in cucumber.

The fruit diameter heterosis showed a range of -31.75 $\left(\mathrm{P}_{3} \times \mathrm{P}_{4}\right)$ to $6.41\left(\mathrm{P}_{5} \times \mathrm{P}_{1}\right)$. Among thirty hybrids, four hybrids expressed positive significant values and sixteen hybrids recorded negative significant values. The maximum values were recorded in $\mathrm{P}_{3} \mathrm{X} \mathrm{P}_{4}$ ($31.75 \%), \mathrm{P}_{3} \mathrm{X}_{6}(31.16 \%)$ as the fruit size was controlled by partial dominance of additive gene action (Table 1). 
Int.J.Curr.Microbiol.App.Sci (2018) 7(3): 3025-3032

Table.1 Standard heterosis for vegetative traits of pumpkin

\begin{tabular}{|c|c|c|c|c|}
\hline Hybrids & $\begin{array}{c}\text { Days to first } \\
\text { Female flowering }\end{array}$ & $\begin{array}{l}\text { Nodes to first } \\
\text { Male flower }\end{array}$ & Sex ratio & $\begin{array}{c}\text { Days to first } \\
\text { harvest }\end{array}$ \\
\hline $\mathbf{P}_{1} \times \mathbf{P}_{2}$ & $-9.03 *$ & 3.48 & -3.45 & 0.97 \\
\hline $\mathbf{P}_{1} \times \mathbf{P}_{3}$ & -6.71 & 2.61 & 0.51 & 1.98 \\
\hline $\mathbf{P}_{1} \times \mathbf{P}_{4}$ & -0.44 & -3.87 & $1.08 * *$ & 1.67 \\
\hline $\mathbf{P}_{1} \times \mathbf{P}_{5}$ & -1.80 & 0.95 & $1.36^{* *}$ & $0.84 *$ \\
\hline $\mathbf{P}_{1} \times \mathbf{P}_{6}$ & 3.82 & -2.53 & 1.93 & 1.46 \\
\hline $\mathbf{P}_{2} \times \mathbf{P}_{1}$ & $2.24^{*}$ & 7.90 & 4.08 & -3.10 \\
\hline $\mathbf{P}_{2} \times \mathbf{P}_{3}$ & 0.96 & 4.03 & 4.02 & -0.78 \\
\hline $\mathbf{P}_{2} \times \mathbf{P}_{4}$ & 1.89 & 7.90 & $2.12 * *$ & -1.36 \\
\hline $\mathbf{P}_{2} \times \mathbf{P}_{5}$ & $5.73 *$ & 31.6 & 4.56 & -3.48 \\
\hline $\mathbf{P}_{2} \times \mathbf{P}_{6}$ & 2.55 & $44.71 * *$ & 3.52 & -2.64 \\
\hline $\mathbf{P}_{3} \times \mathbf{P}_{1}$ & 3.82 & 0.08 & 13.14 & 0.56 \\
\hline $\mathbf{P}_{3} \times \mathbf{P}_{2}$ & 3.18 & -2.69 & 6.74 & 0.42 \\
\hline $\mathbf{P}_{3} \times \mathbf{P}_{4}$ & 5.73 & 10.51 & $9.37 * *$ & 0.86 \\
\hline $\mathbf{P}_{3} \times \mathbf{P}_{5}$ & 0.00 & 7.90 & $7.93 *$ & 1.06 \\
\hline $\mathbf{P}_{3} \times \mathbf{P}_{6}$ & 1.17 & $43.44 * *$ & 5.92 & 1.51 \\
\hline $\mathbf{P}_{4} \times \mathbf{P}_{1}$ & -0.44 & 15.80 & $36.24 * *$ & 0.45 \\
\hline $\mathbf{P}_{4} \times \mathbf{P}_{2}$ & -1.27 & -12.16 & $33.01 * *$ & 4.62 \\
\hline $\mathbf{P}_{4} \times \mathbf{P}_{3}$ & -0.97 & 10.51 & $25.71 * *$ & 1.53 \\
\hline $\mathbf{P}_{4} \times \mathbf{P}_{5}$ & -0.84 & 5.21 & $28.4^{* *}$ & 2.54 \\
\hline $\mathbf{P}_{4} \times \mathbf{P}_{6}$ & 4.67 & -10.74 & $33.49 * *$ & $1.88 *$ \\
\hline $\mathbf{P}_{5} \times \mathbf{P}_{1}$ & 5.22 & 18.64 & $34.31 * *$ & $6.46^{*}$ \\
\hline $\mathbf{P}_{5} \times \mathbf{P}_{2}$ & $1.69 *$ & -2.69 & $12.32 * *$ & 3.25 \\
\hline $\mathbf{P}_{5} \times \mathbf{P}_{3}$ & 3.82 & 17.69 & $11.26 *$ & 1.33 \\
\hline $\mathbf{P}_{5} \times \mathbf{P}_{4}$ & 5.41 & 24.49 & $0.31 * *$ & 0.11 \\
\hline $\mathbf{P}_{5} \times \mathbf{P}_{6}$ & $5.10 * *$ & $21.09 *$ & -6.57 & -0.21 \\
\hline $\mathbf{P}_{6} \times \mathbf{P}_{1}$ & -0.95 & 14.06 & -8.18 & 0.03 \\
\hline $\mathbf{P}_{6} \times \mathbf{P}_{2}$ & 1.05 & $18.4 * *$ & -15.83 & 3.45 \\
\hline $\mathbf{P}_{6} \times \mathbf{P}_{3}$ & 3.82 & $36.89 * *$ & -15.83 & 4.94 \\
\hline $\mathbf{P}_{6} \times \mathbf{P}_{4}$ & -0.32 & 13.19 & $-4.70 * *$ & $6.28 *$ \\
\hline $\mathbf{P}_{6} \times \mathbf{P}_{5}$ & $5.30 * *$ & $34.20 *$ & -10.62 & 5.85 \\
\hline \multicolumn{2}{|c|}{ * Significant at 5 per cent level } & \multicolumn{3}{|c|}{ ** Significant at 1 per cent level } \\
\hline
\end{tabular}


Int.J.Curr.Microbiol.App.Sci (2018) 7(3): 3025-3032

Table.2 Standard heterosis for yield traits of pumpkin

\begin{tabular}{|c|c|c|c|c|c|c|c|}
\hline Hybrids & $\begin{array}{l}\text { Fruit } \\
\text { length }\end{array}$ & $\begin{array}{c}\text { Fruit } \\
\text { diameter }\end{array}$ & $\begin{array}{c}\text { Flesh } \\
\text { thickness }\end{array}$ & $\begin{array}{c}\text { No. of } \\
\text { seeds } \\
\text { per fruit }\end{array}$ & $\begin{array}{l}\text { No. of } \\
\text { fruits } \\
\text { per vine }\end{array}$ & $\begin{array}{c}\text { A verage } \\
\text { fruit } \\
\text { weight }\end{array}$ & $\begin{array}{c}\text { Fruit } \\
\text { Yield } \\
\text { per vine }\end{array}$ \\
\hline $\mathbf{P}_{1} \times \mathbf{P}_{2}$ & $10.04 * *$ & $1.44 * *$ & -2.89 & $3.37 * *$ & $-25.58 * *$ & 164.70 & $139.45 * *$ \\
\hline $\mathbf{P}_{1} \times \mathbf{P}_{3}$ & $9.82 * *$ & $2.62 * *$ & -13.10 & 4.28 & $-17.67^{*}$ & 132.77 & $134.25 * *$ \\
\hline $\mathbf{P}_{1} \times \mathbf{P}_{4}$ & 13.98 & 2.74 & -11.90 & -0.26 & $-62.56 * *$ & 113.08 & $81.50 * *$ \\
\hline $\mathbf{P}_{1} \times \mathbf{P}_{5}$ & $16.64 * *$ & 1.37 & -19.56 & $-3.61 * *$ & $-26.05 * *$ & 113.22 & $206.79 * *$ \\
\hline $\mathbf{P}_{1} \times \mathbf{P}_{6}$ & $14.18 * *$ & 3.97 & $-12.41 *$ & $5.56 \mathrm{~ns}$ & $-35.12 * *$ & 72.29 & $143.35 * *$ \\
\hline $\mathbf{P}_{2} \times \mathbf{P}_{1}$ & $-33.54 * *$ & $-15.92 * *$ & -19.22 & $-20.37 * *$ & $-15.81 * *$ & -56.82 & $-34.25 * *$ \\
\hline $\mathbf{P}_{2} \times \mathbf{P}_{3}$ & $-33.65 * *$ & $-16.10 * *$ & $-19.73 * *$ & $-14.21 * *$ & -8.14 & -49.93 & -11.56 \\
\hline $\mathbf{P}_{2} \times \mathbf{P}_{4}$ & $-33.27 * *$ & $-17.02 * *$ & -13.95 & -16.66 & $-33.26 * *$ & -49.79 & $-9.97 * *$ \\
\hline $\mathbf{P}_{2} \times \mathbf{P}_{5}$ & $-33.19 * *$ & $-15.31 *$ & $-18.03 *$ & $-16.10 * *$ & -5.35 & -43.18 & $-32.66 * *$ \\
\hline $\mathbf{P}_{2} \times \mathbf{P}_{6}$ & $-32.60 * *$ & $-17.53 * *$ & -1.53 & $-22.15 *$ & $-34.42 * *$ & -52.88 & -36.13 \\
\hline $\mathbf{P}_{3} \times \mathbf{P}_{1}$ & $-33.54 * *$ & $-30.87 * *$ & -6.63 & -0.39 & -7.44 & -6.75 & $-0.29 * *$ \\
\hline $\mathbf{P}_{3} \times \mathbf{P}_{2}$ & $-34.41 * *$ & $-30.51 * *$ & $-26.19 * *$ & $0.48 * *$ & -9.30 & -38.40 & 8.96 \\
\hline $\mathbf{P}_{3} \times \mathbf{P}_{4}$ & $-36.83 * *$ & $-31.75 * *$ & $-14.46 *$ & 1.14 & $-31.16 * *$ & -34.60 & $-12.72 * *$ \\
\hline $\mathbf{P}_{3} \times \mathbf{P}_{5}$ & $-37.02 * *$ & $-29.95 * *$ & $-24.32 * *$ & 1.51 & -7.44 & -20.82 & 5.64 \\
\hline $\mathbf{P}_{3} \times \mathbf{P}_{6}$ & $-35.56 * *$ & $-31.16 * *$ & -22.28 & $1.58 * *$ & -12.33 & -31.50 & $12.86 * *$ \\
\hline $\mathbf{P}_{4} \times \mathbf{P}_{1}$ & -11.26 & -5.46 & 0.51 & 4.39 & $-30.93 * *$ & $71.87 *$ & $171.53 * *$ \\
\hline $\mathbf{P}_{4} \times \mathbf{P}_{2}$ & $-12.13 * *$ & $-3.57 * *$ & -8.84 & 18.10 & $-34.65 * *$ & -6.33 & $182.95 * *$ \\
\hline $\mathbf{P}_{4} \times \mathbf{P}_{3}$ & $-16.75 * *$ & $-6.07 * *$ & $-10.37 *$ & 4.60 & $-25.81 * *$ & $-10.27 * *$ & $166.76 * *$ \\
\hline $\mathbf{P}_{4} \times \mathbf{P}_{5}$ & $-12.07 *$ & -1.88 & $-8.50 * *$ & $5.42 * *$ & -1.63 & 53.16 & $167.34 * *$ \\
\hline $\mathbf{P}_{4} \times \mathbf{P}_{6}$ & $-10.72 * *$ & $-4.66 * *$ & -8.84 & $3.85 * *$ & $-25.35 * *$ & 51.05 & $162.86 * *$ \\
\hline $\mathbf{P}_{5} \times \mathbf{P}_{1}$ & $32.91 * *$ & 6.41 & 13.27 & $17.80 * *$ & $-36.28 * *$ & $117.44^{*}$ & $150.29 * *$ \\
\hline $\mathbf{P}_{5} \times \mathbf{P}_{2}$ & $0.74 * *$ & $5.90 *$ & $-5.10 *$ & $4.00 * *$ & $-6.28 * *$ & -9.00 & $2.75 * *$ \\
\hline $\mathbf{P}_{5} \times \mathbf{P}_{3}$ & $-2.20 * *$ & $1.77 * *$ & $-8.33 * *$ & -2.67 & 23.72 & $-13.36 * *$ & -3.76 \\
\hline $\mathbf{P}_{5} \times \mathbf{P}_{4}$ & $0.63 *$ & -2.11 & $-22.45 * *$ & $7.38 * *$ & -6.28 & -9.70 & $-1.73 * *$ \\
\hline $\mathbf{P}_{5} \times \mathbf{P}_{6}$ & $-2.64 * *$ & $-2.44 *$ & -13.44 & $-2.67 *$ & 0.93 & -12.94 & $-9.97 * *$ \\
\hline $\mathbf{P}_{6} \times \mathbf{P}_{1}$ & $1.05 * *$ & -1.50 & $-11.56 *$ & 1.02 & $-25.35 * *$ & $-0.28^{*}$ & $-13.73 * *$ \\
\hline $\mathbf{P}_{6} \times \mathbf{P}_{2}$ & $1.52 * *$ & $-8.90 * *$ & 5.44 & $12.19 *$ & $-16.28 * *$ & 3.94 & 25.58 \\
\hline $\mathbf{P}_{6} \times \mathbf{P}_{3}$ & $-15.05 * *$ & $-4.35 * *$ & 12.24 & $12.97 * *$ & -15.35 & 20.25 & $19.22 * *$ \\
\hline $\mathbf{P}_{6} \times \mathbf{P}_{4}$ & $-21.86 * *$ & $-11.53 * *$ & 20.41 & $10.99 * *$ & $-15.81 * *$ & -18.57 & $20.66 * *$ \\
\hline $\mathbf{P}_{6} \times \mathbf{P}_{5}$ & $-21.67 * *$ & $-9.07 *$ & 11.22 & $12.22 *$ & -20.93 & -2.11 & $42.20 * *$ \\
\hline
\end{tabular}

* Significant at 5 per cent level

** Significant at 1 per cent level 
Table.3 Standard heterosis for qualitative traits of pumpkin

\begin{tabular}{|c|c|c|c|}
\hline Hybrids & $\begin{array}{c}\text { Total soluble } \\
\text { solids }\end{array}$ & $\begin{array}{c}\text { Beta carotene } \\
\text { content }\end{array}$ & $\begin{array}{c}\text { Dry matter } \\
\text { content }\end{array}$ \\
\hline $\mathbf{P}_{1} \times \mathbf{P}_{2}$ & $-25.50 * *$ & 0.69 & $29.87 * *$ \\
\hline $\mathbf{P}_{1} \times \mathbf{P}_{3}$ & $-29.20 * *$ & $0.69 * *$ & $20.78 * *$ \\
\hline $\mathbf{P}_{1} \times \mathbf{P}_{4}$ & $-17.99 *$ & 1.39 & $22.08 * *$ \\
\hline $\mathbf{P}_{1} \times \mathbf{P}_{5}$ & $-27.30 * *$ & 13.19 & $29.87 * *$ \\
\hline $\mathbf{P}_{1} \times \mathbf{P}_{6}$ & $-31.50 * *$ & $11.11 * *$ & 15.58 \\
\hline $\mathbf{P}_{2} \times \mathbf{P}_{1}$ & $-17.32 * *$ & 17.36 & $33.77 * *$ \\
\hline $\mathbf{P}_{2} \times \mathbf{P}_{3}$ & $-18.11 * *$ & $29.17 * *$ & $18.18 *$ \\
\hline $\mathbf{P}_{2} \times \mathbf{P}_{4}$ & -15.41 & $19.44 *$ & $24.68 * *$ \\
\hline $\mathbf{P}_{2} \times \mathbf{P}_{5}$ & $-17.15 * *$ & 5.56 & $18.83 * *$ \\
\hline $\mathbf{P}_{2} \times \mathbf{P}_{6}$ & $-14.07 * *$ & $20.14 * *$ & $31.17 *$ \\
\hline $\mathbf{P}_{3} \times \mathbf{P}_{1}$ & $-9.92 * *$ & $28.47 * *$ & $0.00 * *$ \\
\hline $\mathbf{P}_{3} \times \mathbf{P}_{2}$ & $3.42 * *$ & $29.17 * *$ & $-2.60 *$ \\
\hline $\mathbf{P}_{3} \times \mathbf{P}_{4}$ & 2.30 & $25.69 * *$ & $23.38 * *$ \\
\hline $\mathbf{P}_{3} \times \mathbf{P}_{5}$ & -7.06 & $25.00 *$ & -1.30 \\
\hline $\mathbf{P}_{3} \times \mathbf{P}_{6}$ & $-6.89 * *$ & $16.67 * *$ & 10.39 \\
\hline $\mathbf{P}_{4} \times \mathbf{P}_{1}$ & $4.76 *$ & 9.03 & $18.83 * *$ \\
\hline $\mathbf{P}_{4} \times \mathbf{P}_{2}$ & 5.77 & $2.78 *$ & $6.49 * *$ \\
\hline $\mathbf{P}_{4} \times \mathbf{P}_{3}$ & 2.30 & $5.56 * *$ & $5.19 * *$ \\
\hline $\mathbf{P}_{4} \times \mathbf{P}_{5}$ & 0.67 & 9.72 & 11.69 \\
\hline $\mathbf{P}_{4} \times \mathbf{P}_{6}$ & $0.78 * *$ & $10.42 * *$ & 11.04 \\
\hline $\mathbf{P}_{5} \times \mathbf{P}_{1}$ & $-18.16 * *$ & -7.64 & $9.09 * *$ \\
\hline $\mathbf{P}_{5} \times \mathbf{P}_{2}$ & $-6.45 * *$ & 0.69 & $5.19 * *$ \\
\hline $\mathbf{P}_{5} \times \mathbf{P}_{3}$ & 5.94 & $0.00 *$ & -2.60 \\
\hline $\mathbf{P}_{5} \times \mathbf{P}_{4}$ & 3.70 & 4.17 & 0.65 \\
\hline $\mathbf{P}_{5} \times \mathbf{P}_{6}$ & $8.46 *$ & -2.08 & $1.30 *$ \\
\hline $\mathbf{P}_{6} \times \mathbf{P}_{1}$ & $-21.41 * *$ & $19.44 * *$ & -14.94 \\
\hline $\mathbf{P}_{6} \times \mathbf{P}_{2}$ & $-25.45 * *$ & $24.31 * *$ & $-16.88 *$ \\
\hline $\mathbf{P}_{6} \times \mathbf{P}_{3}$ & $-14.74 * *$ & $18.06 * *$ & -17.53 \\
\hline $\mathbf{P}_{6} \times \mathbf{P}_{4}$ & $-20.18 * *$ & $25.69 * *$ & -13.64 \\
\hline $\mathbf{P}_{6} \times \mathbf{P}_{5}$ & $-19.51 *$ & 18.06 & $-15.58 *$ \\
\hline
\end{tabular}


Smaller sized fruits crossed with bigger sized fruits resulted in decrease in fruit size of the hybrid. This was in consonance with the finding of Muthaiah et al., (2017) in ridge gourd.

The flesh thickness heterosis showed a range of $-26.19\left(\mathrm{P}_{3} \times \mathrm{P}_{2}\right)$ to $20.41\left(\mathrm{P}_{6} \times \mathrm{P}_{4}\right)$. Among thirty hybrids, six hybrids expressed positively non-significant heterosis while twelve hybrids recorded negative significant standard heterosis. The highest heterotic expression was recorded in $\mathrm{P}_{3} \times \mathrm{P}_{2}(-26.19 \%)$ followed by $\mathrm{P}_{3} \times \mathrm{P}_{5}(-24.32 \%)$. This present result is in accordance with Rana et al., (2016) in pumpkin.

The number of seeds per fruit heterosis showed a range of from $-22.15\left(\mathrm{P}_{2} \times \mathrm{P}_{6}\right)$ to $18.10\left(\mathrm{P}_{4} \times \mathrm{P}_{2}\right)$. Among thirty hybrids, twelve hybrids recorded positive standard heterosis and six hybrids recorded significant standard heterosis. The highest heterotic expression was recorded in $\mathrm{P}_{2} \times \mathrm{P}_{6}$ (-22.15per cent) followed by $\mathrm{P}_{2} \times \mathrm{P}_{1}$ (-20.37 per cent). Similar result was obtained by Muthaiah et al., (2017) in ridge gourd.

The extent of fruit weight heterosis ranged between -56.82 $\left(\mathrm{P}_{2} \times \mathrm{P}_{1}\right)$ and $164.7\left(\mathrm{P}_{1} \times \mathrm{P}_{2}\right)$ per cent. Only two hybrids recorded positively significant value. The cross $\mathrm{P}_{2} \times \mathrm{P}_{4}$ $(71.87 \%)$ and $\mathrm{P}_{5} \times \mathrm{P}_{1}(117.44 \%)$ exhibited significant and positive standard heterosis for average fruit weight. The fruit size was governed by partial dominance of additive gene action. This was in accordance with the results of Gvozdanovic Varga et al., (2011) in water melon.

The number of fruits per vine heterosis ranged from -62.56 $\left(\mathrm{P}_{1} \mathrm{x} \mathrm{P}_{4}\right)$ to $23.72\left(\mathrm{P}_{5} \times \mathrm{P}_{3}\right)$. Among thirty hybrids, there was no positive heterotic value, whereas eighteen hybrids recorded negatively significant standard heterosis. The lowest values recorded in $\mathrm{P}_{2} \times$
$\mathrm{P}_{1}(-15.81 \%), \mathrm{P}_{6} \times \mathrm{P}_{4}(-15.81 \%), \mathrm{P}_{6} \times \mathrm{P}_{2}(-$ $167.28 \%), \mathrm{P}_{1} \times \mathrm{P}_{3}(-17.67 \%)$ and the highest effect was observed in $\mathrm{P}_{1} \times \mathrm{P}_{4}(-62.56 \%), \mathrm{P}_{5} \times$ $\mathrm{P}_{1}(-36.28 \%)$ and $\mathrm{P}_{1} \times \mathrm{P}_{6}(-35.12 \%)$. Similar results were obtained by Kumar et al., (2010) in cucumber.

The extent of heterosis over standard variety ranged between $-36.13\left(\mathrm{P}_{2} \times \mathrm{P}_{6}\right)$ and 206.79 per cent $\left(\mathrm{P}_{1} \times \mathrm{P}_{5}\right)$. Among thirty hybrids, sixteen hybrids exhibited positive and eight hybrids exhibited negative significant standard heterotic value. The crosses $\mathrm{P}_{1} \times \mathrm{P}_{5}$ (206.79\%), $\mathrm{P}_{4} \times \mathrm{P}_{2} \quad(182.95 \%), \mathrm{P}_{4} \times \mathrm{P}_{1}$ (171.53\%), $\mathrm{P}_{4} \times \mathrm{P}_{5}(167.34 \%)$, and $\mathrm{P}_{4} \times \mathrm{P}_{3}$ (166.76\%) exhibited significant and positive standard heterosis for fruit yield per plant due to heterotic expression of additive gene action. Muthaiah et al., (2017) in ridge gourd was also reported similar results (Table 2).

\section{Quality traits}

Total soluble solids are important for sweetness of pumpkin, increases the quality and marketability. Significant and positive standard heterosis for total soluble solids was exhibited by the crosses $\mathrm{P}_{4} \times \mathrm{P}_{1}(4.76 \%)$ and $\mathrm{P}_{5} \times \mathrm{P}_{6}(8.46 \%)$ by non-additive partial dominance gene action. This result confirmed the findings of Rana et al., (2016) in pumpkin (Table 3).

Beta carotene is one of the important traits for quality of fruit. Orange colour of pumpkin fruit is due to beta carotene. Significant and positive standard heterosis for beta carotene content was exhibited by the crosses $\mathrm{P}_{2} \times \mathrm{P}_{3}$ (29.17\%), $\mathrm{P}_{3} \times \mathrm{P}_{2}(29.17 \%)$, and $\mathrm{P}_{3} \times \mathrm{P}_{1}$ $(28.47 \%)$, due to the non-additive over dominance gene action. This is in agreement with the results of Nisha and Veeraragavathatham (2014) in pumpkin.

The highest standard heterosis values were recorded in $\mathrm{P}_{2} \times \mathrm{P}_{1} \quad(33.77 \%), \mathrm{P}_{1} \times \mathrm{P}_{2}$ 
(29.87\%), $\mathrm{P}_{1} \times \mathrm{P}_{5}(29.87 \%)$, and $\mathrm{P}_{2} \times \mathrm{P}_{4}$ $(24.68 \%)$ crosses for dry matter content and the expression was due to over dominance of non-additive gene action. This present results are in accordance with the Aravindakumar et al., (2005) in muskmelon.

The cross combinations $\mathrm{P}_{1} \times \mathrm{P}_{2}, \mathrm{P}_{1} \times \mathrm{P}_{4}, \mathrm{P}_{1} \mathrm{x}$ $\mathrm{P}_{5}, \mathrm{P}_{1} \times \mathrm{P}_{6}$ and $\mathrm{P}_{4} \times \mathrm{P}_{1}$ exhibited positive standard heterosis traits like days to first female flowering, fruit length, fruit diameter and yield per plant. It could be used for higher yield with bigger sized fruits. The smaller sized fruits with negative heterosis were observed in $\mathrm{P}_{2} \times \mathrm{P}_{1}(-34.25 \%)$ and $\mathrm{P}_{2} \times \mathrm{P}_{5}$ ($32.66 \%$ ) crosses.

\section{References}

Anupam, A., Randhir, K., Amit, K. and Singh, H. K. 2017. Estimation of gene action and heterosis in bottle gourd (Lagenaria siceraria Mol. Standl.). Environment and Ecology, 35 (2A): 936-944.

Doijode, S.D. 1994. Correlation studies in pumpkin. Haryana J. Hort. Sci., 11(1-2): 42-45.

Doijode, S.D. and Sulladmath, U.V. 1983. Genetic variability and correlation studies in pumpkin. Mysore J. Agric. Sci., 20 (1): $59-61$.

Gvozdanovic Varga, J., Vasić, M., Milić, D., and Červenski, J. 2011. Diallel cross analysis for fruit traits in watermelon. Genetika, 43(1): 163-174.

Hedau, N.K. and Sirohi, P.S. 2006. A diallel studies in ridge gourd [Luffa acutangula (Roxb) L.]. Orissa J.Hort., 34(2):6-12.

Kumar, J., Munshi, A.D., Kema, R., and Sureja, A.K. 2010. Studies on Heterosis in slicing cucumber. Indian J. Hort., 67(2): 197-201.

Muthaiah, K., Gasti, V. D., Mallesh, S. and Nagaraju, K. 2017. Heterosis studies for earliness and yield related traits in ridge gourd [Luffa acutangula (L.) Roxb.]. Int.J.Curr. Microbiol. App. Sci, 6 (6): 2656-2661.

Nisha, S. K and Veeraragavathatham, D. 2014. Heterosis and combining ability for fruit yield and its component traits in pumpkin (Cucurbita moschata Duch. ex Poir.). Adv. in Applied Res. 6 (2): 158-162.

Rana, M. S., Rasu, M. G., Islam, A. K. M. A. and Hossain, M. M. 2016. Diallel Analysis of Quality and Yield Contributing Traits of Pumpkin (Cucurbita moschata Duch. ex Poir.). The Agriculturists, 14(1): 15-32.

Saxena, M., and Chander, P.G. 2015. Indian horticulture database-2014, IG printer Pvt. Ltd. New Delhi, p: 279

Turner, J.H. 1953. A study of heterosis in upland cotton. I. Yield of hybrids compared with varieties. Agron. J., 45: 484-486.

\section{How to cite this article:}

Marxmathi, P., V. Krishnamoorthy and Thankaraj, P. 2018. Studies on Heterosis in Pumpkin (Cucurbita moschata Duch. ex. Poir). Int.J.Curr.Microbiol.App.Sci. 7(03): 3025-3032. doi: https://doi.org/10.20546/ijcmas.2018.703.351 